\title{
The influence of $(1,3)-(1,6)-\beta-D$-glucan on the production results of sows and their offspring
}

\author{
A. Szuba-Trznadel ${ }^{1,4}$, A. Rząsa ${ }^{2}$, R. Lira ${ }^{3}$ and B. Fuchs ${ }^{1}$ \\ Wroclaw University of Environmental and Life Sciences \\ 1 Department of Animal Nutrition and Feed Management, J. Chełmońskiego 38D, 51-630 Wroclaw, Poland \\ ${ }^{2}$ Department of Immunology, Pathophysiology and Veterinary Prevention, C.K. Norwida 31, 50-375 Wroclaw, Poland \\ ${ }^{3}$ LIRA Feed Manufacture, Chłapowskiego 36, 64-010 Krzywiń, Poland
}

KEY WORDS: sows, piglets, health, growth performance, glucan

Received: 3 October 2012

Revised: 12 May 2014

Accepted: 21 August 2014

${ }^{4}$ Corresponding author:

e-mail: anna.szuba-trznadel@up.wroc.pl

\begin{abstract}
The aim of the study was to assess the production results and health status of sows and their offspring modulated by supplementation of selected amounts of $(1,3)-(1,6)-\beta$-D-glucan. It was added in doses of 0,100 , 200 , or $300 \mathrm{ppm}$ to the feed mixtures for sows from day 80 of gestation and for their offspring from day 10 to 45 of life. Production results were estimated based on: daily gains, average feed intake and conversion ratio, and mortality. Blood, colostrum and milk samples were collected to estimate immunological stimulation based on the levels of total protein and its fractions, and on haptoglobin. Twelve representative pigs (aged 45 days) were slaughtered and the small intestine was dissected. The obtained data suggest that the best effect on health status and production results was obtained when doses of 200 and $300 \mathrm{ppm}$ of $\beta$-glucans were used.
\end{abstract}

\section{Introduction}

The total withdrawal of antibiotic growth promoters from pig diets in January 2006 prompted a search for the best alternatives to them. Among these alternatives, the (1,3)-(1,6)- $\beta$-D-glucan isolated from brewer's yeast, Saccharomyces cerevisiae, seems to be a very interesting option. Many studies have demonstrated its beneficial influence on the health of pigs and their production performance (Dritz et al., 1995; Eicher et al., 2006; Li et al., 2006), but some studies reported no benefits (Hiss and Sauerwein, 2003). The last cited authors admitted, however, that the investigations were fragmentary and did not involve the entire production cycle.

The aim of the present study was to assess the production results and health status of sows and their offspring modulated by supplementation of $(1,3)-(1,6)-\beta-D$-glucan in experimental doses.

\section{Material and methods}

The investigation was performed on 40 crossbred sows (Polish Large White $\times$ Polish Landrace) and their 411 offspring at a commercial farm. The animals were subject to routine care and veterinary treatment performed on the farm. On the second day after parturition, the litters were standardized according to size and body weight; the smallest and weakest piglets were culled according to farm technology. In a farrowing unit, piglets were housed with their dams in triple slatted farrowing pens. Iron in the form of an intramuscular injection was given to piglets twice: on days 2 and 19 of life. During the suckling period, from day 10 of life, the piglets had free access to solid feed (Prestarter). After weaning (on day 21 of life) the piglets were moved to a nursery unit and kept in grouped pens for 30 weaners. They were fed the same feed up to day 45 of life. 
The animals were assigned to four different groups: I - control (standard feed used at the farm), and experimental, which were provided additionally with purified $(>80 \%)(1,3)-(1,6)-\beta$-D-glucan supplemented at a level of: II -100 , III -200 , IV -300 ppm, respectively (Table 1). Feed was prepared according to NRC (1998) recommendations. The source of $\beta$-glucan was Betamune ${ }^{\circledR}$. The sows were provided with the experimental feed from day 80 of gestation and were moved into the farrowing building 10 days before expected parturition. The daily dose of the mixture for sows at the farrowing unit was gradually increased up to day 7 after parturition, next they were fed ad libitum to day 19 , and for the last two days of lactation, the daily feed dose was limited to $4.5 \mathrm{~kg}$.
The following production data were collected during the course of the experiment: body weight (BW) of sows on the first and last (weaning) day of stay in a farrowing pen, and of piglets on days 2, 21 (weaning) and 45 (the end of experiment) of life, feed intake (FI) of sows (in the farrowing building) and piglets (in the nursing building), piglet losses, finally, daily gains (DG) and the feed conversion ratio (FCR) were calculated. To estimate health status, samples of blood, colostrum and milk were obtained from 10 randomly selected sows and piglets in every group. Blood was collected into serum tubes from the vena jugularis externa from sows on days 2 and 21 of lactation; from piglets on days 2, 21 and 45 of life. Colostrum samples were taken on day 2, and milk samples, on day 21 after parturition (after

Table 1. Chemical composition and nutritive value of mixtures for sows and of prestarter mixtures for piglets

\begin{tabular}{|c|c|c|c|c|c|c|c|c|}
\hline \multirow{3}{*}{ Indices } & \multicolumn{4}{|c|}{ Sows } & \multicolumn{4}{|c|}{ Piglets } \\
\hline & \multicolumn{8}{|c|}{ Experimental groups } \\
\hline & 1 & II & III & IV & 1 & II & III & IV \\
\hline$(1,3)-(1,6)-\beta$-D-glucan (80\% purity), ppm & 0 & 100 & 200 & 300 & 0 & 100 & 200 & 300 \\
\hline \multicolumn{9}{|l|}{ Ingredients, \% } \\
\hline wheat & & 36.96 & & & & 66.175 & & \\
\hline barley & & 18.00 & & & & & & \\
\hline triticale & & 15.00 & & & & & & \\
\hline wheat bran & & 9.00 & & & & & & \\
\hline soyabean meal, HP46 & & 10.00 & & & & & & \\
\hline soyabean meal, HP300 & & & & & & 10.00 & & \\
\hline fish meal, $72 \%$ & & 2.00 & & & & 7.00 & & \\
\hline dried whey & & & & & & 10.00 & & \\
\hline rape seeds & & 4.00 & & & & & & \\
\hline rape oil & & 1.50 & & & & 3.00 & & \\
\hline fodder chalk & & 1.00 & & & & & & \\
\hline 1-calcium phosphate & & 0.80 & & & & 0.80 & & \\
\hline calcium formate & & 0.20 & & & & 0.30 & & \\
\hline salt & & 0.50 & & & & 0.30 & & \\
\hline lysine $\mathrm{HCl}$ & & 0.28 & & & & 0.54 & & \\
\hline DL-methionine, $98 \%$ & & 0.05 & & & & 0.21 & & \\
\hline L-threonine, $98 \%$ & & 0.12 & & & & 0.19 & & \\
\hline Optimax ${ }^{1}$ & & 0.50 & & & & 0.50 & & \\
\hline Optimin ZMC² & & 0.04 & & & & & & \\
\hline Nordocid & & & & & & 0.50 & & \\
\hline Ferkel Kirch Mende & & & & & & 0.02 & & \\
\hline $\mathrm{ZnO}, 78 \%$ & & & & & & 0.315 & & \\
\hline Lidermix-Optazyme $0.1 \%$ enzyme & & & & & & 0.10 & & \\
\hline Myco-AD-AZ & & 0.05 & & & & 0.05 & & \\
\hline \multicolumn{9}{|l|}{ Nutritional value } \\
\hline metabolizable energy, $\mathrm{MJ} \cdot \mathrm{kg}^{-1}$ & & 13.12 & & & & 14.30 & & \\
\hline crude protein, $\%$ & & 15.38 & & & & 19.00 & & \\
\hline $\mathrm{Ca}, \%$ & & 0.87 & & & & 0.81 & & \\
\hline $\mathrm{P}$ total, $\%$ & & 0.66 & & & & 0.74 & & \\
\hline lysine, $\%$ & & 0.98 & & & & 1.54 & & \\
\hline methionine, $\%$ & & 0.29 & & & & 0.54 & & \\
\hline methionine and cystine, $\%$ & & 0.60 & & & & 0.89 & & \\
\hline tryptophan, \% & & 0.19 & & & & 0.23 & & \\
\hline threonine, $\%$ & & 0.65 & & & & 0.90 & & \\
\hline
\end{tabular}

${ }^{1}$ composition of mineral-vitamin mixture - Optimax $0.5 \%$ in IU: vit. $A 4000$, vit. $D_{3} 400 ; \mathrm{g} \cdot \mathrm{kg}^{-1}$ : vit. E 16 , vit. $B_{1} 0.20$, vit. $B_{2} 0.80$, biotin 0.05 , vit. $B_{6} 0.60$, vit. $B_{12} 0.004$, vit. C 8.00 , vit. K 0.40 , niacin 4.00 , folic acid 1.00 , calcium panthotenate 3.00 , choline $60.00, \mathrm{Mg} 2.00, \mathrm{Mn} 10.00$, Zn 16.00, Co 0.10, Se 0.06, Fe 25.00, J 0.20, Cu 3.00, Ca 258.37, antioxidant 0.1, Cl 15.26

${ }^{2}$ Optimin - mineral preparation: organic form of, $\mathrm{g} \cdot \mathrm{kg}^{-1}: \mathrm{Zn} 80, \mathrm{Mn} 40$ and $\mathrm{Cu} 20$, supplemented by soya protein 
injection of $2 \mathrm{ml}$ of oxytocin). Total protein (TP) and its fractions were estimated in serum as well as in colostrum and milk whey. The level of TP was estimated using the BCA test, and its fractions, using filter paper electrophoresis. Additionally, the guaiacol (peroxidase) test was applied in serum to measure the haptoglobin (Hp) level (Jones and Mould, 1984).

On the $45^{\text {th }}$ day of life, 12 representative healthy barrows ( 3 from every group) were randomly chosen and slaughtered in order to dissect the small intestine. Six 2-cm-long scraps of intestine (intenstinum tепие) were cut $20 \mathrm{~cm}$ below the duodenum. The segments were gently rinsed in buffered saline water and fixed in buffered glutarate aldehyde and formalin. The tissue scraps were examined using a scanning electron microscope (Leo Zeiss $435 \mathrm{VP}$, Oberkochen, Germany) and an Axio Imager fluorescence microscope (Zeiss, Oberkochen, Germany). The following features were taken into consideration during the histological analysis: location and shape of intestinal villi, both number and intensity of intestine glandular membrane damage, presence and activity of glandular cells, as well as integrity of the basement membrane.

The collected data were evaluated statistically. Treatment comparisons were made by analysis of variance procedures for a completely randomized design using the STATISTICA (2011). Differences between the means were determined by the post-hoc Tukey's test at the 0.05 and 0.01 levels.

\section{Results}

The changes in BW and FI of sows at the farrowing unit are presented in Table 2. The average BW before parturition was similar in all groups $(247.3 \mathrm{~kg})$. Moreover, there were no statistical differences in BW losses during lactation, the mean values amounted to $42.7 \mathrm{~kg}$. Comparable losses were noted in groups I, II and III, and were: $17.8 \%$, $17.3 \%$ and $17.4 \%$ of initial BW, respectively. The lowest weight loss was recorded in group IV, $16.5 \%$. The average FI for sows was $7.9 \mathrm{~kg}$ per day. The lowest FI was noted in group I and this result was statistically different compared with the highest FI, found in group III $(P \leq 0.05)$.

The initial number and BW of piglets after standardization was similar in all groups (Table 2). The same tendency was observed on weaning day, however, at the end of the experiment statistically confirmed differences were found in BW between group I (control) and two experimental groups, III and IV. The observed differences in BW and DG at the nursing unit were related to FCR. The highest
Table 2. Production results of sows and their piglets

\begin{tabular}{|c|c|c|c|c|c|c|}
\hline \multirow{2}{*}{ Indices } & & \multicolumn{4}{|c|}{ Experimental groups } & \multirow{2}{*}{ SEM } \\
\hline & & I & $\|$ & III & IV & \\
\hline \multicolumn{7}{|l|}{ Body weight of sows } \\
\hline on 2 days before & $x$ & 241 & 248 & 245 & 255 & 7.74 \\
\hline parturition, $\mathrm{kg} / \mathrm{head}$ & \pm & 26.91 & 30.46 & 27.27 & 9.16 & \\
\hline on $21^{\text {st }}$ day of & $x$ & 198 & 205 & 202 & 213 & 7.25 \\
\hline lactation, $\mathrm{kg} / \mathrm{head}$ & \pm & 23.29 & 30.14 & 24.23 & 9.79 & \\
\hline \multicolumn{7}{|l|}{ Feed intake of mixture } \\
\hline by sows during & $x$ & $7.69^{\mathrm{a}}$ & 7.95 & $8.13^{b}$ & 7.97 & 0.10 \\
\hline lactation, kg/sow/day & \pm & 0.32 & 0.30 & 0.27 & 0.32 & \\
\hline \multicolumn{7}{|l|}{ No. of piglets } \\
\hline \multirow[t]{2}{*}{ on the $2^{\text {nd }}$ day } & $x$ & 99 & 107 & 99 & 100 & 0.42 \\
\hline & \pm & 1.65 & 0.82 & 1.25 & 1.32 & \\
\hline \multirow[t]{2}{*}{ on the $21^{\text {st }}$ day } & $x$ & 88 & 93 & 95 & 96 & 0.35 \\
\hline & \pm & 1.45 & 0.85 & 0.97 & 1.17 & \\
\hline \multirow[t]{2}{*}{ on the $45^{\text {th }}$ day } & $x$ & & & & & 0.41 \\
\hline & \pm & 1.47 & 1.20 & 1.07 & 1.17 & \\
\hline \multicolumn{7}{|l|}{ Losses of piglets, head $\%$} \\
\hline \multirow[t]{2}{*}{$2^{\text {nd }}$ to $21^{\text {st }}$ day } & $x$ & 11 & 14 & 4 & 4 & \\
\hline & & 11.00 & 12.84 & 4.04 & 4.00 & \\
\hline \multirow[t]{2}{*}{$22^{\text {nd }}$ to $45^{\text {th }}$ day } & $x$ & 6 & 2 & 1 & - & \\
\hline & & 6.82 & 2.15 & 1.05 & - & \\
\hline \multicolumn{7}{|l|}{ Body weight of piglets, $\mathrm{kg}$} \\
\hline \multirow[t]{2}{*}{ on the $2^{\text {nd }}$ day } & $x$ & 1.58 & 1.64 & 1.66 & 1.63 & 0.06 \\
\hline & \pm & 0.25 & 0.23 & 0.12 & 0.12 & \\
\hline \multirow[t]{2}{*}{ on the $21^{\text {st }}$ day } & $x$ & 6.73 & 7.20 & 6.98 & 6.95 & 0.22 \\
\hline & \pm & 0.80 & 0.70 & 0.70 & 0.67 & \\
\hline \multirow[t]{2}{*}{ on the $45^{\text {th }}$ day } & $x$ & $14.27^{\mathrm{a}}$ & 14.63 & $15.11^{b}$ & $15.05^{b}$ & 0.22 \\
\hline & \pm & 0.62 & 0.58 & 0.62 & 0.60 & \\
\hline \multicolumn{7}{|l|}{ Daily gain of piglets, $\mathrm{g}$} \\
\hline \multirow[t]{2}{*}{$2^{\text {nd }}$ to $21^{\text {st }}$ day } & $x$ & 270.992 & 292.73 & 280.34 & 280.17 & 12.06 \\
\hline & \pm & 46.52 & 37.22 & 36.89 & 38.25 & \\
\hline \multirow[t]{2}{*}{$22^{\text {nd }}$ to $45^{\text {th }}$ day } & $x$ & 314.133 & 309.73 & 338.37 & 337.47 & 11.75 \\
\hline & \pm & 39.38 & 38.68 & 34.41 & 29.87 & \\
\hline \multirow[t]{2}{*}{$2^{\text {nd }}$ to $45^{\text {th }}$ day } & $x$ & $295.07^{\text {a }}$ & 302.22 & $312.73^{b}$ & $312.15^{b}$ & 5.45 \\
\hline & \pm & 17.33 & 16.33 & 16.18 & 14.54 & \\
\hline \multicolumn{7}{|c|}{ Feed conversion ratio, $\mathrm{kg} \cdot \mathrm{kg}^{-1}$} \\
\hline \multirow[t]{2}{*}{$21^{\text {st }}$ to $45^{\text {th }}$ day } & $x$ & $1.48^{\mathrm{A}}$ & $1.42^{\mathrm{B}}$ & $1.43^{\mathrm{B}}$ & $1.41^{\mathrm{B}}$ & 0.01 \\
\hline & \pm & 0.02 & 0.02 & 0.01 & 0.01 & \\
\hline
\end{tabular}

$\mathrm{A}, \mathrm{B}, \mathrm{a,b}$ means with different superscripts within a row are significantly different at $P \leq 0.01$ and $P \leq 0.05$, respectively

FCR was in the control group, and this result differed significantly from the other groups at $P \leq 0.01$.

The changes in total protein and its fractions in sow serum during lactation are shown in Table 3. On day 2 after parturition, the lowest concentration of TP, albumin and globulin was observed in group II and there were statistically confirmed differences at $P \leq 0.01$ compared with group III (the highest content). Statistically meaningful differences appeared in different globulin fractions between the experimental groups. 
Table 3. Total protein and its fraction in sows serum

\begin{tabular}{|c|c|c|c|c|c|c|}
\hline \multirow{2}{*}{\multicolumn{2}{|c|}{ Specification, $\left.g \cdot\right|^{-1}$}} & \multicolumn{4}{|c|}{ Experimental groups } & \multirow{2}{*}{ SEM } \\
\hline & & I & ॥ & III & IV & \\
\hline \multicolumn{7}{|c|}{$2^{\text {nd }}$ day after parturition } \\
\hline Total protein & $x$ & $\begin{array}{r}59.26 \\
6.65\end{array}$ & $\begin{array}{c}50.76^{\mathrm{A}} \\
9.37\end{array}$ & $\begin{array}{c}70.03^{B} \\
8.96\end{array}$ & $\begin{array}{r}62.63 \\
9.78\end{array}$ & 4.11 \\
\hline Albumins & $\begin{array}{l}x \\
\pm\end{array}$ & $\begin{array}{r}26.07 \\
3.40\end{array}$ & $\begin{array}{r}24.55 \\
5.99\end{array}$ & $\begin{array}{r}31.02 \\
7.95\end{array}$ & $\begin{array}{r}28.41 \\
4.52\end{array}$ & 2.29 \\
\hline a-globulin & $x$ & $\begin{array}{l}8.40^{A} \\
1.88\end{array}$ & $\begin{array}{l}9.58^{\mathrm{a}} \\
2.17\end{array}$ & $\begin{array}{c}13.21^{\mathrm{Bb}} \\
2.84\end{array}$ & $\begin{array}{l}9.21^{\mathrm{a}} \\
1.90\end{array}$ & 1.08 \\
\hline$\beta$-globulin & $x$ & $\begin{array}{r}11.35 \\
2.27\end{array}$ & $\begin{array}{l}9.33^{A} \\
1.18\end{array}$ & $\begin{array}{c}12.69^{\mathrm{Bb}} \\
1.53\end{array}$ & $\begin{array}{l}9.94^{\mathrm{a}} \\
1.31\end{array}$ & 0.77 \\
\hline Y-globulin & $x$ & $\begin{array}{c}13.94^{\mathrm{A}} \\
2.36\end{array}$ & $\begin{array}{l}7.58^{B} \\
1.07\end{array}$ & $\begin{array}{c}13.23^{\mathrm{A}} \\
4.58\end{array}$ & $\begin{array}{c}13.24^{\mathrm{A}} \\
2.42\end{array}$ & 1.45 \\
\hline \multicolumn{7}{|c|}{$21^{\text {st }}$ day after parturition } \\
\hline Total protein & $x$ & $\begin{array}{r}65.33 \\
8.54\end{array}$ & $\begin{array}{r}75.22 \\
7.87\end{array}$ & $\begin{array}{c}76.40^{\mathrm{a}} \\
5.16\end{array}$ & $\begin{array}{l}63.02^{b} \\
10.38\end{array}$ & 3.68 \\
\hline Albumins & y & $\begin{array}{r}29.39 \\
5.68\end{array}$ & $\begin{array}{r}31.77 \\
2.71\end{array}$ & $\begin{array}{r}31.12 \\
3.81\end{array}$ & $\begin{array}{r}26.18 \\
4.11\end{array}$ & 1.69 \\
\hline a-globulin & $\mathbf{I}$ & $\begin{array}{r}10.54 \\
1.44\end{array}$ & $\begin{array}{r}12.08 \\
1.67\end{array}$ & $\begin{array}{r}12.34 \\
2.21\end{array}$ & $\begin{array}{r}12.56 \\
1.25\end{array}$ & 0.67 \\
\hline$\beta$-globulin & $x$ & $\begin{array}{r}11.89 \\
1.98\end{array}$ & $\begin{array}{r}14.02 \\
2.46\end{array}$ & $\begin{array}{r}13.52 \\
2.32\end{array}$ & $\begin{array}{r}10.93 \\
2.15\end{array}$ & 0.92 \\
\hline Y-globulin & $\hat{ \pm}$ & $\begin{array}{c}13.52^{\mathrm{a}} \\
2.01\end{array}$ & $\begin{array}{r}17.35 \\
3.97\end{array}$ & $\begin{array}{c}19.43^{b} \\
3.60\end{array}$ & $\begin{array}{r}15.67 \\
4.61\end{array}$ & 1.53 \\
\hline
\end{tabular}

A,B,a,b means with different superscripts within a row are significantly different at $P \leq 0.01$ and $P \leq 0.05$, respectively

The mean values of TP on day 21 were more balanced between the groups, however, a significant difference $(P \leq 0.05)$ was noted between groups III and IV. The lowest concentration of $\gamma$-globulin was determined in group I, and this result statistically differed from the highest value observed in group III.

The haptoglobin level in sows (Figure 1) on day 2 of lactation was the highest in group III and statistically differed from the results obtained in the other groups, i.e. at $P \leq 0.01$ in group IV and at $P \leq 0.05$ in groups I and II. At the end of lactation, the Hp content was low and there were no differences among the groups.

The changes in TP and levels of its fractions in piglet serum during the experimental period are

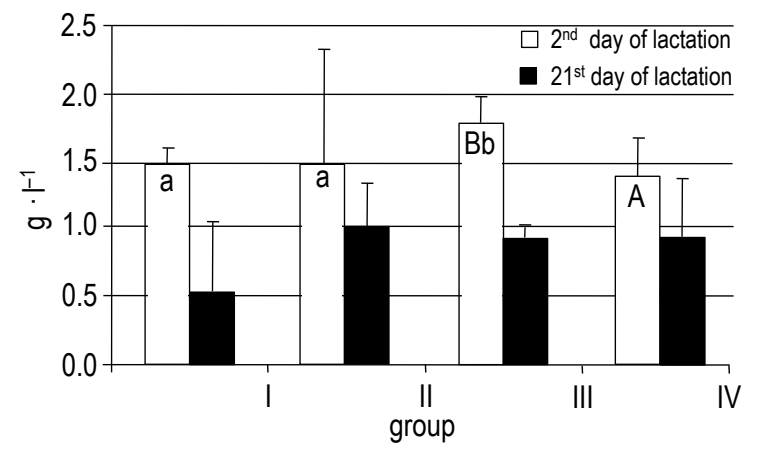

Figure 1. Haptoglobin concentration in sow serum. $a, b-P \leq 0.05$; $A, B-P \leq 0.01$
Table 4. Total protein and its fraction in piglets serum

\begin{tabular}{|c|c|c|c|c|c|c|}
\hline \multirow{2}{*}{\multicolumn{2}{|c|}{ Specification, $g \cdot l^{-1}$}} & \multicolumn{4}{|c|}{ Experimental groups } & \multirow{2}{*}{ SEM } \\
\hline & & \multirow{2}{*}{\multicolumn{2}{|c|}{$\begin{array}{l}\text { on } 2^{\text {nd }} \text { day of life } \\
\end{array}$}} & \multirow[t]{2}{*}{ III } & \multirow[t]{2}{*}{ IV } & \\
\hline & & & & & & \\
\hline Total protein & $\begin{array}{l}x \\
\pm\end{array}$ & $\begin{array}{l}47.43 \\
7.65\end{array}$ & $\begin{array}{l}53.37 \\
14.85\end{array}$ & $\begin{array}{l}40.53^{\mathrm{A}} \\
5.47\end{array}$ & $\begin{array}{l}60.61^{\mathrm{B}} \\
8.52\end{array}$ & 4.50 \\
\hline Albumins & $\begin{array}{l}x \\
\pm\end{array}$ & $\begin{array}{l}14.53 \\
2.98\end{array}$ & $\begin{array}{l}10.71^{\mathrm{A}} \\
2.77\end{array}$ & $\begin{array}{l}12.14 \\
2.20\end{array}$ & $\begin{array}{l}15.85^{\mathrm{B}} \\
2.75\end{array}$ & 1.23 \\
\hline a-globulin & $\begin{array}{l}x \\
\pm\end{array}$ & $\begin{array}{l}6.48^{\mathrm{Aa}} \\
1.77\end{array}$ & $\begin{array}{l}10.17^{\mathrm{B}} \\
1.28\end{array}$ & $\begin{array}{l}9.24^{b} \\
1.76\end{array}$ & $\begin{array}{l}10.29^{\mathrm{B}} \\
2.07\end{array}$ & 0.86 \\
\hline$\beta$-globulin & $\begin{array}{l}x \\
\pm\end{array}$ & $\begin{array}{l}10.23 \\
1.50\end{array}$ & $\begin{array}{l}12.14 \\
4.33\end{array}$ & $\begin{array}{l}8.98 \\
2.11\end{array}$ & $\begin{array}{l}12.46 \\
5.26\end{array}$ & 1.41 \\
\hline Y-globulin & $\begin{array}{l}x \\
\pm\end{array}$ & $\begin{array}{l}16.18 \\
2.66\end{array}$ & $\begin{array}{l}20.35^{A} \\
9.71\end{array}$ & $\begin{array}{l}10.16^{\mathrm{B}} \\
1.66\end{array}$ & $\begin{array}{l}22.02^{A} \\
4.29\end{array}$ & 2.64 \\
\hline & & on $21^{\text {st }}$ & day of life & & & \\
\hline Total protein & $\begin{array}{l}x \\
\pm\end{array}$ & $\begin{array}{r}52.83 \\
3.43\end{array}$ & $\begin{array}{r}52.87 \\
5.36\end{array}$ & $\begin{array}{r}44.82 \\
8.31\end{array}$ & $\begin{array}{r}49.90 \\
4.81\end{array}$ & 2.39 \\
\hline Albumins & $\begin{array}{l}x \\
\pm\end{array}$ & $\begin{array}{c}32.65^{\mathrm{a}} \\
2.34\end{array}$ & $\begin{array}{c}24.26^{b} \\
6.01\end{array}$ & $\begin{array}{c}23.95^{b} \\
7.56\end{array}$ & $\begin{array}{r}28.06 \\
2.98\end{array}$ & 2.28 \\
\hline a- globulin & $\begin{array}{l}x \\
\pm\end{array}$ & $\begin{array}{l}7.88 \\
1.96\end{array}$ & $\begin{array}{r}12.04 \\
5.42\end{array}$ & $\begin{array}{l}8.91 \\
1.68\end{array}$ & $\begin{array}{l}8.86 \\
2.46\end{array}$ & 1.29 \\
\hline$\beta$-globulin & $\begin{array}{l}x \\
\pm\end{array}$ & $\begin{array}{l}7.22^{\mathrm{A}} \\
1.19\end{array}$ & $\begin{array}{c}10.76^{\mathrm{B}} \\
1.38\end{array}$ & $\begin{array}{l}8.74 \\
1.59\end{array}$ & $\begin{array}{l}9.11 \\
2.35\end{array}$ & 0.77 \\
\hline Y-globulin & $\begin{array}{l}x \\
\pm\end{array}$ & $\begin{array}{l}5.10 \\
1.80\end{array}$ & $\begin{array}{l}5.81^{\mathrm{A}} \\
1.03\end{array}$ & $\begin{array}{l}3.23^{\mathrm{B}} \\
1.15\end{array}$ & $\begin{array}{l}3.88 \\
1.15\end{array}$ & 0.61 \\
\hline & & on $45^{\text {th }}$ & day of life & & & \\
\hline Total protein & $\begin{array}{l}x \\
\pm\end{array}$ & $\begin{array}{l}47.43 \\
13.13\end{array}$ & $\begin{array}{r}48.72 \\
6.26\end{array}$ & $\begin{array}{r}41.80 \\
9.29\end{array}$ & $\begin{array}{r}43.65 \\
9.40\end{array}$ & 3.63 \\
\hline albumins & $\begin{array}{l}x \\
\pm\end{array}$ & $\begin{array}{r}23.63 \\
7.50\end{array}$ & $\begin{array}{r}24.16 \\
4.97\end{array}$ & $\begin{array}{r}20.89 \\
5.08\end{array}$ & $\begin{array}{r}23.41 \\
5.65\end{array}$ & 2.13 \\
\hline a-globulin & $\begin{array}{l}x \\
\pm\end{array}$ & $\begin{array}{r}12.34 \\
2.50\end{array}$ & $\begin{array}{r}10.65 \\
0.98\end{array}$ & $\begin{array}{l}9.86 \\
2.30\end{array}$ & $\begin{array}{l}9.82 \\
2.43\end{array}$ & 0.85 \\
\hline$\beta$-globulin & $\begin{array}{l}x \\
\pm\end{array}$ & $\begin{array}{l}7.41 \\
2.62\end{array}$ & $\begin{array}{l}8.21 \\
0.98\end{array}$ & $\begin{array}{l}6.83 \\
1.91\end{array}$ & $\begin{array}{l}6.83 \\
1.00\end{array}$ & 0.66 \\
\hline Y-globulin & $\begin{array}{l}x \\
\pm\end{array}$ & $\begin{array}{l}4.04 \\
1.50\end{array}$ & $\begin{array}{l}5.70^{\mathrm{a}} \\
0.84\end{array}$ & $\begin{array}{l}4.22 \\
1.02 \\
\end{array}$ & $\begin{array}{l}3.59^{b} \\
0.93\end{array}$ & 0.49 \\
\hline
\end{tabular}

$A, B, a, b$ means with different superscripts within a row are significantly different at $P \leq 0.01$ and $P \leq 0.05$, respectively

presented in Table 4. On day 2 of life, the lowest concentration of TP was recorded in group III, and this result differed significantly from the value obtained in group IV $(P \leq 0.01)$. Differentiation among the groups did not increase to the end of the observation period. The mean albumin concentrations were

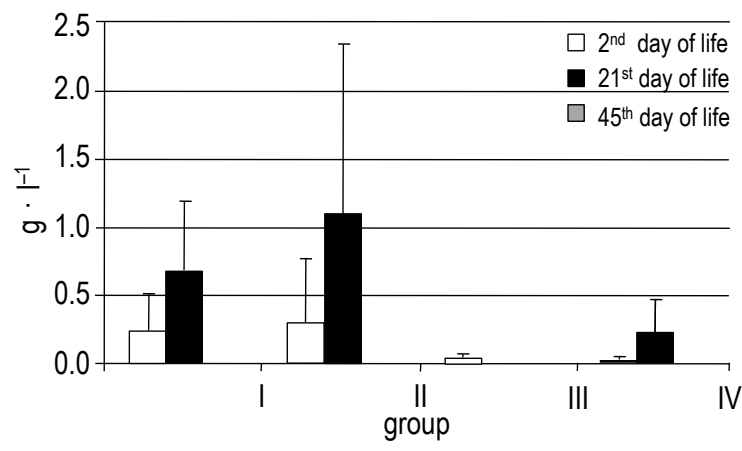

Figure 2. Haptoglobin concentration in piglets serum 


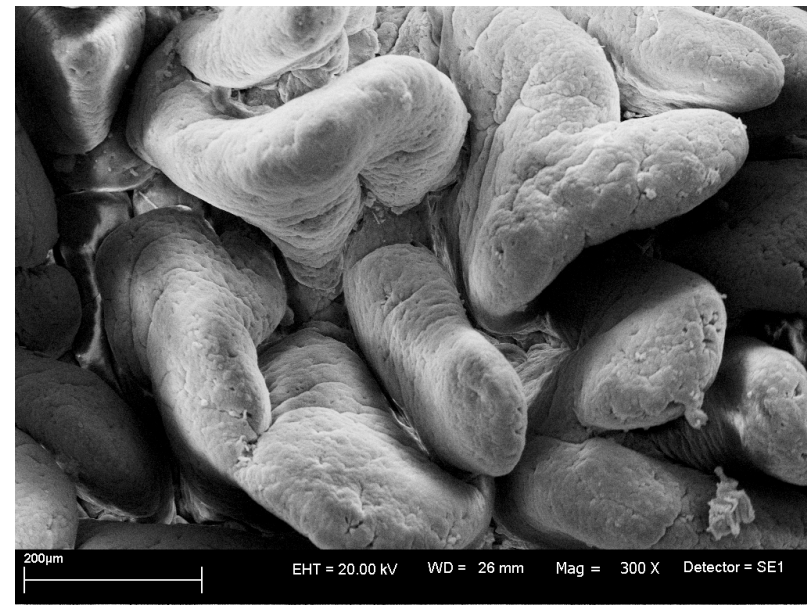

Figure 3. Group I. The general view of the intestine fragment. 300x

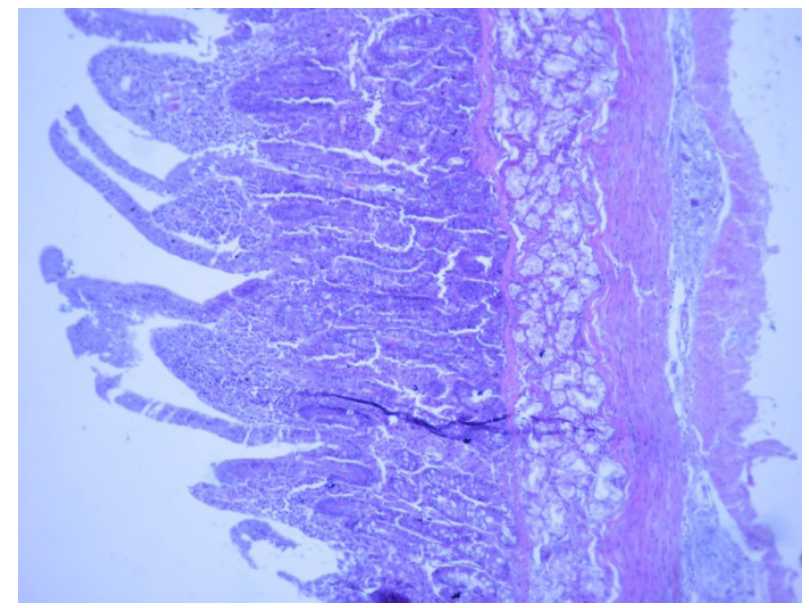

Figure 5. Group II. Transverse cross-section of the intestine fragment. $40 x$

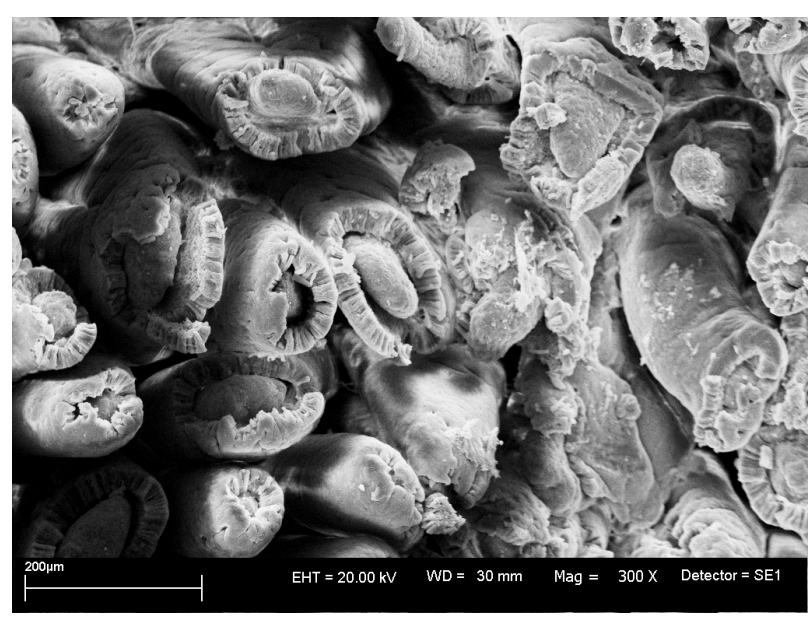

Figure 7. Group III. The general view of the intestine fragment. 300x

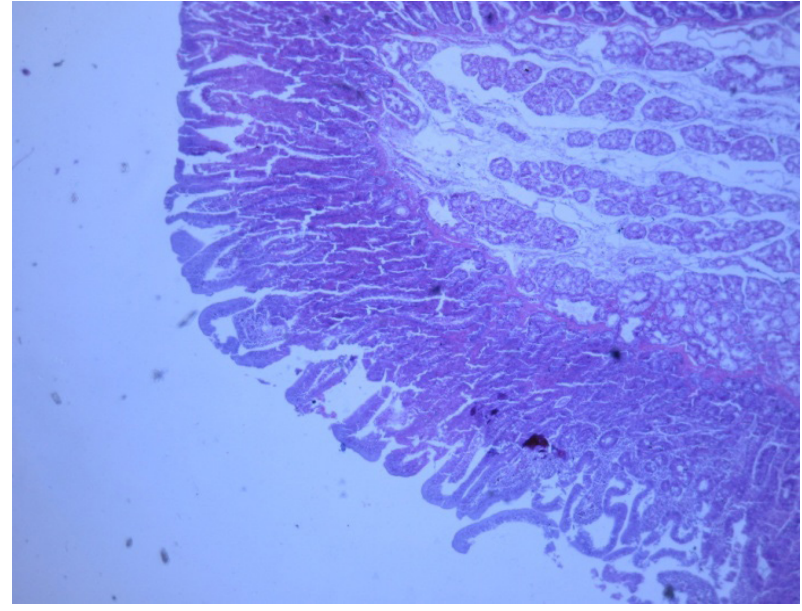

Figure 4. Group II. Transverse cross-section of the intestine fragment. $40 x$

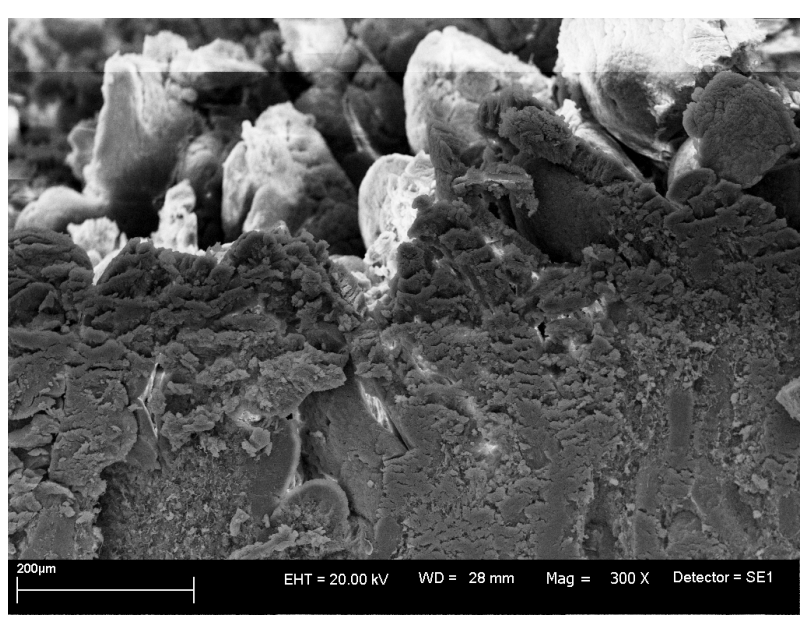

Figure 6. Group II. Transverse cross-section of the intestine fragment - the cross-sections of particular villi are visible. $300 x$

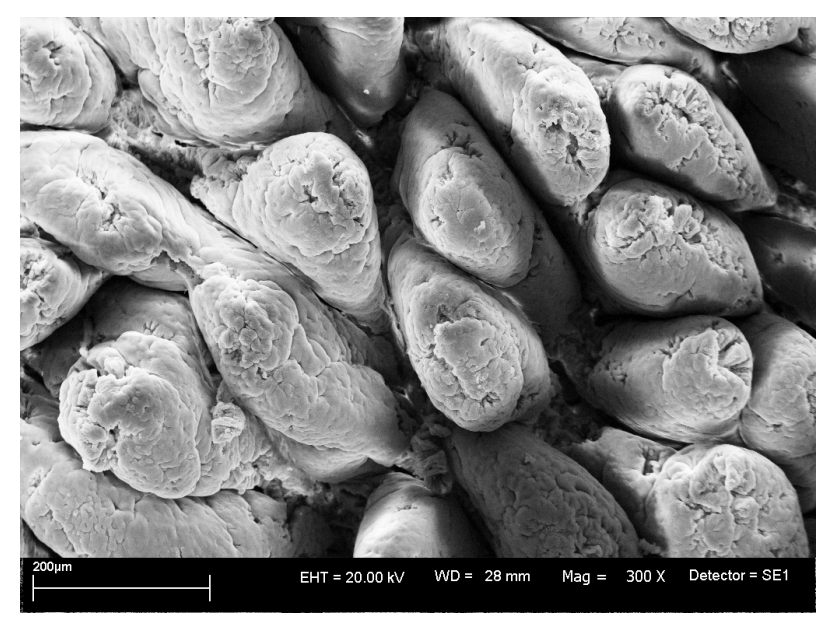

Figure 8. Group IV. The general view of the intestine fragment. 300x 
Table 5. Total protein and its fraction in sows colostrum and milk

\begin{tabular}{|c|c|c|c|c|c|c|}
\hline \multirow{2}{*}{\multicolumn{2}{|c|}{ Specification, $g \cdot l^{-1}$}} & \multicolumn{4}{|c|}{ Experimental groups } & \multirow{2}{*}{ SEM } \\
\hline & & I & $\|$ & III & IV & \\
\hline $\begin{array}{l}\text { Colostrum whey } \\
\text { total protein }\end{array}$ & $\begin{array}{l}x \\
\pm\end{array}$ & $\begin{array}{r}151.74 \\
63.89\end{array}$ & $\begin{array}{r}144.18 \\
47.31\end{array}$ & $\begin{array}{r}118.85 \\
31.69\end{array}$ & $\begin{array}{r}127.09 \\
27.16\end{array}$ & 14.27 \\
\hline albumins & $\begin{array}{l}x \\
\pm\end{array}$ & $\begin{array}{c}16.58^{\mathrm{a}} \\
2.72\end{array}$ & $\begin{array}{l}25.86^{\mathrm{Bb}} \\
10.76\end{array}$ & $\begin{array}{c}17.39^{a} \\
4.99\end{array}$ & $\begin{array}{c}15.31^{\mathrm{A}} \\
5.44\end{array}$ & 2.47 \\
\hline a-globulin & $\begin{array}{l}x \\
\pm\end{array}$ & $\begin{array}{r}20.87 \\
6.51\end{array}$ & $\begin{array}{r}18.96 \\
9.58\end{array}$ & $\begin{array}{r}16.05 \\
4.74\end{array}$ & $\begin{array}{r}17.92 \\
7.81\end{array}$ & 2.31 \\
\hline$\beta$-globulin & $\begin{array}{l}x \\
\pm\end{array}$ & $\begin{array}{l}23.68 \\
13.31\end{array}$ & $\begin{array}{l}27.17 \\
11.38\end{array}$ & $\begin{array}{l}27.12 \\
12.58\end{array}$ & $\begin{array}{r}25.73 \\
9.36\end{array}$ & 3.56 \\
\hline v-globulin & $\begin{array}{l}x \\
\pm\end{array}$ & $\begin{array}{l}72.10 \\
17.46\end{array}$ & $\begin{array}{l}72.20 \\
40.81\end{array}$ & $\begin{array}{l}58.30 \\
18.68\end{array}$ & $\begin{array}{l}68.14 \\
18.40\end{array}$ & 8.15 \\
\hline $\begin{array}{l}\text { Milk whey } \\
\text { total protein }\end{array}$ & $\begin{array}{l}x \\
\pm\end{array}$ & $\begin{array}{r}17.00 \\
3.83\end{array}$ & $\begin{array}{r}18.81 \\
1.37\end{array}$ & $\begin{array}{r}18.57 \\
2.18\end{array}$ & $\begin{array}{r}17.24 \\
2.39\end{array}$ & 0.83 \\
\hline albumins & $\begin{array}{l}x \\
\pm\end{array}$ & $\begin{array}{l}3.90 \\
1.69\end{array}$ & $\begin{array}{l}3.07 \\
1.36\end{array}$ & $\begin{array}{l}4.34 \\
1.34\end{array}$ & $\begin{array}{l}3.62 \\
0.85\end{array}$ & 0.43 \\
\hline a-globulin & $\begin{array}{l}x \\
\pm\end{array}$ & $\begin{array}{l}4.34^{\mathrm{a}} \\
2.27\end{array}$ & $\begin{array}{l}2.73^{b} \\
0.73\end{array}$ & $\begin{array}{l}2.73^{b} \\
0.46\end{array}$ & $\begin{array}{l}3.89 \\
0.69\end{array}$ & 0.45 \\
\hline$\beta$-globulin & $\begin{array}{l}x \\
\pm\end{array}$ & $\begin{array}{l}4.71^{\mathrm{a}} \\
2.93\end{array}$ & $\begin{array}{l}2.62 \\
1.8\end{array}$ & $\begin{array}{l}2.36^{b} \\
1.09\end{array}$ & $\begin{array}{l}4.59^{\mathrm{a}} \\
1.13\end{array}$ & 0.65 \\
\hline$\gamma$-globulin & $\begin{array}{l}x \\
\pm \\
\end{array}$ & $\begin{array}{l}4.05^{\mathrm{A}} \\
2.75\end{array}$ & $\begin{array}{c}10.43^{\mathrm{B}} \\
2.07 \\
\end{array}$ & $\begin{array}{l}9.09^{B} \\
2.02\end{array}$ & $\begin{array}{l}5.13^{\mathrm{A}} \\
1.10 \\
\end{array}$ & 1.06 \\
\hline
\end{tabular}

$A, B ; a, b$ means with different superscripts within a row are significantly different at $P \leq 0.01$ and $P \leq 0.05$, respectively

subject to change throughout the entire experimental period. Statistical differences were noted on day 2 between groups IV and II $(\mathrm{P} \leq 0.01)$ and on day 21 between groups I and II, III $(\mathrm{P} \leq 0.05)$. Some statistically significant differences between the groups were found in the globulin fractions depending on the day they were determined, i.e. on day 2 in the $\alpha$ - and $\gamma$-globulin fractions, on day 21 in the $\beta$ - and $\gamma$-globulin fractions, and on day 45 only in the $\gamma$-globulin fraction.

The changes in the Hp concentration in piglet serum are presented in Figure 2. On day 2, the mean values of this index equalled $0.14 \mathrm{~g} \cdot \mathrm{1}^{-1}$. On day 21 , the highest concentration $\left(1.09 \mathrm{~g} \cdot 1^{-1}\right)$ was recorded in group II, but there were no significant differences compared with the other groups. On day 45, Hp was not detected in any of the groups.

The comparison of TP and its fractions in colostrum and milk is presented in Table 5. Very high diversity in colostrum TP was noted among groups, however, it was not confirmed statistically; similar observations in the results concerning milk were not found. Significant differences were recorded in the colostrum albumin fraction at the $P \leq 0.01$ level between groups II and IV, and at the $P \leq 0.05$ level, between groups II and I, III. Statistical differences were also present among milk globulin fractions. The greatest differences at the $P \leq 0.01$ level were noted between groups II and III as well as between groups I and IV.

Regarding the morphology of glandular epithelium, no losses were noted in group II (Figures 4 and 5), while they were considerable in other groups. The analysed intestinal surface showed a reduced degree of destruction due to the death of the animals, which caused disconnection of the gland stroma from its epithelium. Further analysis showed that animals from groups III and IV had a higher intestinal villi (villi intestinales) density (Figures 6, 7 and 8) compared with the control group, which did not receive dietary $\beta$-glucan (Figure 3 ). The control group villi had a clearly oval, finger-like shape and were located in great proximity to each other. The animals in the other groups did not reveal such features. No lobar damages in the intestinal villi were observed in group II (Figure 7). The animals from groups III and IV were characterized by the highest number of glandular cells (Figures 7 and 8).

\section{Discussion}

No differences in body weight (BW) losses of sows were observed among dietary treatment groups during lactation. Similar observations with a yeast supplement to sow diets were made by Veum et al. (1995) and Jurgens et al. (1997). In the present study, however, $\beta$-glucan supplementation affected sow feed intake. Although Jurgens et al. (1997) did not find a significant effect on feed intake, the sows fed yeast treatments tended to consume less feed over the 3-week lactation period compared with those fed the control diet.

On day 2 after parturition, the total protein (TP) concentration in sow serum was low, but such a decrease could be explained by immunoglobulin transfer to colostrum. A relationship can be observed between the highest serum TP level in the group of sows receiving $200 \mathrm{ppm}$ of $\beta$-glucan and the lowest TP value in their colostrum. A physiological increase in this parameter was marked in all groups at the end of lactation. The present observations are similar to those noted by Machado-Neto et al. (1987). Just after parturition, no distinct influence on the examined sow serum globulin fractions of the additive was noted. Such a tendency could be observed at the end of lactation. The mean values of globulin content were the lowest in the control group. The same, expected tendency was noted in case of $\gamma$-globulin content in milk. 
These data indicate that the changes in haptoglobin (Hp) concentration in sow serum seem to be dependent on physiological status and are similar to those described by other authors (Verheyen et al., 2007; Hiss-Pesch et al., 2011).

The data from this experiment are consistent with the results reported by Jurgens et al. (1997), who revealed that yeast treatment increased $\gamma$-globulin fractions in milk. Additionally, one can notice the greater variability in all globulin fractions in control sows compared with the other groups. This effect could be considered an expected, positive influence of $\beta$-glucan supplementation to sow diets.

There were no statistically confirmed differences in piglet BW during the suckling period. The studies of Veum et al. (1995) and Jurgens et al. (1997) support these findings. In the present study, it was difficult to evaluate the influence of the investigated additives on piglet rearing results because of the technological culling carried out at the farm. It can be concluded that the lowest losses were found in groups when 200 or $300 \mathrm{ppm}$ of $\beta$-glucan was added to the diet during the suckling period. Satisfactory piglet rearing results after supplementing pregnant and lactating sows with yeast were reported by Fuchs et al. (2007), who noted a distinct influence of the investigated additives on piglet daily gain (DG) during the entire experimental period. The same tendency was described by Eicher et al. (2006) and Li et al. (2006). It is worth emphasizing that different results are obtained by different researchers using various amounts of $\beta$-glucan. Li et al. (2006) reported higher DG in pigs fed $50 \mathrm{ppm}$ of $\beta$-glucan, in contrast to Hiss and Sauerwein (2003), who used 150 to $300 \mathrm{ppm}$ of $\beta$-glucan and obtained marginal benefits on pig performance. A higher dose of $\beta$-glucan was used by Schoenherr et al. (1994), who confirmed the beneficial influence of this dose.

Looking at TP and its fractions in piglet serum on day 2 of life, it is difficult to find a distinct influence of the investigated additives. The average TP level is similar to that reported by Machado-Neto et al. (1987).

These data indicate that the changes in the Hp concentration in piglet serum during the entire experimental period are similar to those suggested by Piñeiro et al. (2009) as good health indicators. This can be confirmed in the presented study by the dynamics of changes in the $\gamma$-globulin fraction. Several studies investigated the action of yeast cell wall components on the immune function of weaning pigs. Reduced Hp concentration was also reported by Dritz et al. (1995) in $\beta$-glucan-fed pigs.
Taking into account the secretory function of the analysed intestinal segment, the most beneficial results, including the morphology of beaker cells, were in the groups where 200 or $300 \mathrm{ppm}$ of $\beta$-glucan was used. The integrity of the glandular epithelium in terms of the high density of glandular epithelium cells is a dominant feature of small intestine protection against local pathogenic infections. Application of $\beta$-glucans (200-300 ppm in feed) could affect its protection against infections. Similarly, an increase in the villus height/crypt depth ratio was observed in the studies conducted by Pelicano et al. (2005) and Gao et al. (2008) in broiler chickens fed yeast when compared with control birds. These results agree with those from a study carried out by Shen et al. (2009) where yeast supplementation for nursery pigs improved jejunal villus height and the villus height/crypt depth ratio. Other studies, however, have shown that histological evaluation indicated no effect of yeast and cell wall product on villus height, crypt depth and villus height/crypt depth ratio (White et al., 2002; Van der Peet-Schwering et al., 2007). In the present study, as regards the integrity of the entire intestinal villus, the most profitable situation was observed in the case of animals in the group where $100 \mathrm{ppm}$ of $\beta$-glucan was added to the diet.

There was no observed distinct influence of the tested additives on BW changes in sows and piglets. Nonetheless, supplementation of $\beta$-glucan (at the 200-300 ppm level) exhibited a beneficial effect on increasing the $\gamma$-globulin content of sow milk and improved the post-weaning feed conversion ratio and daily gain of piglets.

\section{Conclusions}

The obtained results show that $\beta$-glucan can directly or indirectly influence animal health and performance and could be used as a tool for improving the efficiency of pig production. The observed lower immunological stimulation in animals can influence higher production results because of greater stability of homeostasis.

\section{References}

Dritz S.S., Shi J., Kielian T.L., Goodband R.D., Nelssen J.L., Tokach M.D., Chengappa M.M., Smith J.E., Blecha F., 1995. Influence of dietary beta-glucan on growth performance, nonspecific immunity, and resistance to Streptococcus suis infection in weanling pigs. J. Anim. Sci. 73, 3341-3350

Eicher S.D., McKee C.A., Carroll J.A., Pajor E.A., 2006. Supplemental vitamin $C$ and yeast cell wall $\beta$-glucan as growth enhancers in newborn pigs and as immunomodulators after an endotoxin challenge after weaning. J. Anim. Sci. 84, 2352-2360 
Fuchs B., Rząsa A., Szuba-Trznadel A., Haremza D., 2007. Brewery yeast product B.T. given to pregnant and lactating sows as factor influencing on productivity and piglets health (in Polish). Acta Sci. Pol., Zoot. 6(4), 17-28

Gao J., Zhang H.J., Yu S.H., Wu S.G., Yoon I., Quigley J., Gao Y.P., Qi G.H., 2008. Effects of yeast culture in broiler diets on performance and immunomodulatory function. Poultry Sci. 87, 1377-1384

Hiss S., Sauerwein H., 2003. Influence of dietary b-glucan on growth performance, lymphocyte proliferation, specific immune response and haptoglobulin plasma concentrations in pigs. J. Anim. Physiol. Anim. Nutr. 87, 2-11

Hiss-Pesch S., Daniel F., Dunkelberg-Denk S., Mielenz M., Sauerwein H., 2011. Transfer of maternal haptoglobin to suckling piglets. Vet. Immunol. Immunopathol. 144, 104-110

Jones G.E., Mould D.L., 1984. Adaptation of the guaiacol (peroxidase) test for haptoglobins to a microtitration plate system. Rev. Vet. Sci. 37, 87-92

Jurgens M.H., Rikabi R.A., Zimmerman D.R., 1997. The effect of dietary active dry yeast supplement of performance of sows during gestation-lactation and their pigs. J. Anim. Sci. 75, 593-597

Li J., Li D.F., Xing J.J., Cheng Z.B., Lai C.H., 2006. Effects of ß-glucan extracted from Saccharomyces cerevisiae on growth performance, and immunological and somatotropic responses of pigs challenged with Escherichia coli lipopolysaccharide. J. Anim. Sci. 84, 2374-2381

Machado-Neto R., Graves C.N., Curtis S.E., 1987. Immunoglobulins in piglets from sows heat-stressed prepartum. J. Anim. Sci. $65,445-455$

NRC, 1998. Nutrient Requirements of Swine. 10 th revised Edition. National Academy Press. Washington, DC
Pelicano E.R.L., Souza P.A., Souza H.B.A., Figueiredo D.F., Boiago M.M., Carvalho S.R., Bordon V.F., 2005. Intestinal mucosa development in broiler chickens fed natural growth promotors. Braz. J. Poultry Sci. 7(4), 221-229

Piñeiro C., Piñeiro M., Morales J., Andrés M., Lorenzo E., del Pozo M., Alava M.A., Lampreave F., 2009. Pig-MAP and haptoglobin concentration reference values in swine from commercial farms. Vet. J. 179, 78-84

Schoenherr W.D., Pollmann D.S., Coalson J.A., 1994. Titration of MacroGard-S on growth performance of nursery pigs. J. Anim. Sci. 72, Suppl. 2, 57 (Abstr.)

Shen Y.B., Piao X.S., Kim S.W., Wang L., Liu P., Yoon I., Zhen Y.G., 2009. Effects of yeast culture supplementation on growth performance, intestinal health, and immune response of nursery pigs. J. Anim. Sci. 87, 2614-2624

STATISTICA, 2011. Data Analysis Software System. Statsoft Inc., Version 9.1 (www.statsoft.com)

Van der Peet-Schwering C.M.C., Jansman A.J.M., Smidt H., Yoon I., 2007. Effects of yeast culture on performance, gut integrity, and blood cell composition of weanling pigs. J. Anim. Sci. 85, 3099-3109

Veum T.L., Reyes J., Ellersieck M., 1995. Effect of supplemental yeast culture in sow gestation and lactation diets on apparent nutrient digestibilities and reproductive performance through one reproductive cycle. J. Anim. Sci. 73, 1741-1745

Verheyen A.J.M., Maes D.D.D., Mateusen B., Deprez P., Janssens G.P.J., de Lange L., Counotte G., 2007. Serum biochemical reference values for gestating and lactating sows. Vet. J. 174, 92-98

White L.A., Newman M.C., Cromwell G.L., Lindemann M.D., 2002. Brewers dried yeast as a source of mannan oligosaccharides for weanling pigs. J. Anim. Sci. 80, 2619-2628 